\title{
The Perturbation of Material Density in F(R) Modified Gravity of Polynomial Exponential Form
}

\author{
Vo Van On* \\ Group of Computational Physics and Simulation of Advanced Materials, Faculty of Natural Sciences , \\ Thu Dau Mot University, Binh Duong province, Vietnam
}

\begin{abstract}
This paper investigates the linear perturbation of material density of universe in $f(R)$ modified gravity of polynomial exponential form on the scale of distance below the cosmic horizon (sub-horizon). The results show that the model for the evolution of universe is slightly different from that in the $\Lambda$ CDM standard cosmological model. These can be used to show the difference of this modified gravitational model with the $\Lambda$ CMD standard cosmological model and other cosmological models. We also investigate the ration of $\Psi / \Phi$ and $\mathrm{G}_{\mathrm{eff}} / \mathrm{G}_{\mathrm{N}}$ in the model and show that they are within allowable limits of experiments.
\end{abstract}

Keywords: Linear perturbation; modified gravity; polynomial exponential form; material density of universe.

DOI: $10.7176 /$ APTA/78-05

Publication date:June $30^{\text {th }} 2019$

\section{Introduction}

In 1998, scientists have verified that our universe is accelerating from many observational data [1, 2, 3, 4]. In order to provide an explanation for this accelerated expansion, scientists have come up with quite a few approaches listed in two predominant instructions $[5,6]$. In the first class, some specific forms of matter such as the quintessence model $[7,8,9,10,11,12,13]$, k-essence [14, 15] and the Chaplygin gas [16] are causing this expansion. In the second class, the change of Einstein's gravity on cosmological distances as $f(R)$ modified gravities (see Refs.[17] for reviews) is causing the expansion.

We can test modified models of gravities of $f(R)$ searching at background level and also at the growth of the cosmic structures. When searching the $f(R)$ model at the background level, we can find realistic models that have sequentially the radiation, matter and DE eras very close to the $\Lambda$ CDM one. While many realistic $\mathrm{f}(\mathrm{R})$ models have been constructed, it should be regarded that these models could differ in the growth of material density perturbations although levels of background structure are comparable.

In this paper, we investigate the evolution of material perturbation on cosmic time in a small class of $f(R)$ modified gravity, which called $f(R)$ modified gravity of exponential- polynomial form. The results suggest that these evolutionary aspects can be used to show the difference of this modified gravitational model with the standard cosmological model of $\Lambda \mathrm{CMD}$ and other cosmological models. This article is structured as follows: in section 2, we present cosmological perturbation in the $\Lambda \mathrm{CDM}$ standard universe model; in Section 3 , we consider perturbations of material density in $\mathrm{f}(\mathrm{R})$ modified gravities; in section 4, we investigate the evolution of material perturbation in $\mathrm{f}(\mathrm{R})$ modified gravity of polynomial exponential form; in section 5 and section 6 , we evaluate the ratios $\Psi / \Phi$ and Geff / GN, section 7 is the conclusion of the paper.

\section{Cosmological perturbation in the $\Lambda \mathrm{CDM}$ standard cosmological model}

The metric of perturbed FLRW space- time in longitudinal gauge as follows [18]

$$
d s^{2}=-(1+2 \Phi) d t^{2}+a(t)^{2}(1-2 \Psi)\left(d r^{2}+r^{2} d \theta^{2}+r^{2} \sin ^{2} \theta d \phi^{2}\right)
$$

In the matter dominated era, the non-zero components of energy - momentum tensor are

$$
T_{0}^{0}=-\rho_{m}-\delta \rho_{m}, T_{i}^{0}=-\rho_{m} \partial_{i} v
$$

here $\rho \mathrm{m}$ and $\delta \rho \mathrm{m}$ are the material density and its fluctuation, respectively. $v$ is the velocity of scalar perturbation. From the Einstein equations, we obtain the differential equation for the commoving density perturbation

$$
\delta=\frac{\delta \rho_{m}}{\rho_{m}}+3 H a v
$$


Fourier transformation of density perturbation is

$$
\delta_{k}(t)=\int \frac{d^{3} x}{(2 \pi)^{3 / 2}} \delta(t, \stackrel{\mathrm{r}}{x}) e^{\substack{i k \mathrm{r} \\ \mathrm{r}_{\mathrm{r}}}}
$$

here $\mathrm{k}$ is the sign of a co-moving wavenumber, in the following sections, is written as $\delta$ for simplicity. The evolution of $\delta$ in Fourier space is given by the equation

$$
\ddot{\delta}+2 H \dot{\delta}-4 \pi G_{N} \rho_{m} \delta=0
$$

in matter dominated era, the equation becomes

$$
\ddot{\delta}+\frac{4}{3 t} \dot{\delta}-\frac{2}{3 t^{2}} \delta=0 \text {. }
$$

The equation has two independent solutions $t 2 / 3$ and $t-1$, we only note the growth mode $t^{2 / 3}$

$$
\delta_{k}(t)=\delta_{0 k}\left(\frac{t}{t_{0}}\right)^{2 / 3},
$$

here is an initial value, it is $\delta_{k}\left(t_{0}\right)$.

\section{The perturbation of material density in $f(R)$ modified gravity}

In the $\mathrm{f}(\mathrm{R})$ modified gravity, the action has the following form $[18,19]$

$$
S=\int d^{4} x \sqrt{-g}\left[\frac{1}{16 \pi G_{N}} f(R)+L_{m}\right]
$$

Here GN is Newtonian constant and $L_{m}$ is material Lagrangian.

When $f(R)=R-2 \Lambda$, we return the $\Lambda$ CDM standard cosmological model. Below we consider Lagrangian without cosmological constant and $\mathrm{f}(\mathrm{R})$ vanishes when $\mathrm{R}=0$.

From (9), modified Einstein's equations are as follows:

here

$$
\begin{gathered}
F R_{\mu \nu}-\frac{1}{2} f g_{\mu v}+\left(g_{\mu v} \mathrm{~W}-\nabla_{\mu} \nabla_{v}\right) F=8 \pi G_{N} T_{\mu \nu} \\
F \equiv \frac{d f}{d R} .
\end{gathered}
$$

For like - dust matter, the background equations are

$$
\begin{gathered}
3 F H^{2}=\frac{1}{2}(F R-f)-3 H \dot{F}+8 \pi G_{N} \rho_{m}, \\
-2 F \dot{H}=\ddot{F}-H \dot{F}+8 \pi G_{N} \rho_{m}, \\
\dot{\rho}_{m}+3 H \rho_{m}=0 .
\end{gathered}
$$

here the dot directs the derivative with respective to the cosmic time.

The differential equation describes the density perturbation in the sub horizon region is

$$
\ddot{\delta}+2 H \dot{\delta}-4 \pi G_{e f f} \rho_{m} \delta=0
$$

here

$$
G_{e f f}=\frac{1}{1+F_{R}} \frac{1+4 \frac{k^{2}}{a^{2}} \frac{F_{R R}}{1+F_{R}}}{1+3 \frac{k^{2}}{a^{2}} \frac{F_{R R}}{1+F_{R}}} G_{N} .
$$


with

$$
F_{R} \equiv \frac{d(\mathrm{f}-\mathrm{R})}{d R} ; \quad F_{R R} \equiv \frac{d^{2}(f-R)}{d R^{2}}
$$

\section{Cosmological perturbation in $f(R)$ modified gravity of polynomial exponential form}

In this section we research the evolution of material perturbation in $f(R)$ modified gravity of polynomial exponential form. The action $f(R)$ has the form $[20,21,22]$

$$
f(R)=R+a+\frac{\alpha}{R^{m}}\left(1+b R^{2}+c R^{3}\right) e^{-\beta R^{n}},
$$

with $\alpha>0, \beta>0 ; m=n=1 ; a=-2 \Lambda ; b=c=1$

Expanding approximately $\mathrm{f}(\mathrm{R})$ and with hypothesis that $\beta R<<1$

We have $\quad e^{-\beta R}=1-\beta R$,

$$
\begin{aligned}
& F_{R}=\alpha\left[-3 \beta R^{2}-\frac{1}{R^{2}}-2(\beta-1) R+1\right], \\
& F_{R R}=2 \alpha\left[-\beta+\frac{1}{R^{3}}-3 \beta R+1\right] .
\end{aligned}
$$

In the case, the effective gravitational constant has the form as follows

with

$$
\begin{gathered}
G_{e f f}=\frac{1}{1+F_{R}} \frac{1+4 \frac{k^{2}}{a^{2} R} m}{1+3 \frac{k^{2}}{a^{2} R} m} G_{N}, \\
m=\frac{R F_{R R}}{F_{R}+1},
\end{gathered}
$$

$$
m=\frac{R F_{R R}}{1+F_{R}}=\frac{2 \alpha\left[3 \beta R^{4}+(\beta-1) R^{3}-1\right]}{\alpha+3 \alpha \beta R^{4}+2 \alpha(\beta-1) R^{3}-(\alpha+1) R^{2}}
$$

We investigate two critical cases

4.1. The case of $\frac{k^{2}}{a^{2} R} m>>1$

In this case, we have $G_{e f f} \approx \frac{1}{1+F_{R}} G_{N} \cdot \frac{4}{3}$, the model returns to the Brans - Dicke model with $\omega_{B D}=0$ [23]. In fact, the case $\frac{k^{2}}{a^{2} R} m>>1$ can be done in sub-horizon approximate $\mathrm{k} \gg \mathrm{aH}$ as long as $\mathrm{m}$ is not very smaller unit.

The equation (14) can be written in the following form after change the variable

$$
\delta^{\prime \prime}+\left(\frac{1}{2}-\frac{3}{2} \omega_{e f f}\right) \delta^{\prime}-\frac{3}{2}\left[\frac{1+4 \frac{k^{2}}{a^{2} R} m}{1+3 \frac{k^{2}}{a^{2} R} m} G_{N}\right] \Omega_{m} \delta=0
$$

here

$$
\mathrm{N}=\operatorname{lna} ; \text { ' }=\frac{d}{d N}=\frac{1}{H} d t
$$

and

$$
\omega_{e f f}=-1-\frac{2}{3} \frac{H^{\prime}}{H} ; \quad \Omega_{m}=\frac{\rho_{m}}{3 F H^{2}} ; \quad \xi=\frac{k^{2}}{a^{2} R} m ; \quad m=\frac{R F_{R R}}{1+F_{R}}
$$

With the condition $\frac{k^{2}}{a^{2} R} m>>1$, the equation (23) becomes

$$
\delta^{\prime \prime}+\left(\frac{1}{2}-\frac{3}{2} \omega_{e f f}\right) \delta^{\prime}-2 G_{N} \Omega_{m} \delta=0 .
$$

In the matter dominated era 


$$
\omega_{e f f}=-\frac{m}{1+m} \quad \Omega_{m}=1-\frac{m(7+10 m)}{2(1+m)^{2}} .
$$

We choose the solution in the following form

$$
\delta=c_{+} a^{n_{+}}+c_{-} a^{n_{-}}
$$

Solving (26), we have the solution

$$
n_{ \pm}= \pm \frac{\sqrt{-(m+1)^{2}\left[16\left(8 m^{2}+3 m-2\right)-(4 m+1)^{2}\right]}+4 m^{2}+5 m+1}{4(m+1)^{2}} \text {. }
$$

A solution of the growth mode is $\delta_{m}=c_{+} a^{n_{+}}$,

here

$$
n_{+}=\frac{\sqrt{-(m+1)^{2}\left[16\left(8 m^{2}+3 m-2\right)-(4 m+1)^{2}\right]}+4 m^{2}+5 m+1}{4(m+1)^{2}} \text {. }
$$

In matter dominated era, the scale factor $\mathrm{a}(\mathrm{t})$ depends on cosmic time $\mathrm{t}$ in the form

$$
a(t) \propto t^{2 / 3},
$$

so the growth mode of material perturbation depends on cosmic time in the form

$$
\delta=c_{+} t^{\frac{2}{3} n_{+}}
$$

with $\mathrm{n}+$ like in the expression(30).

Figure 1 shows the growth mode of cosmological perturbation on the scale factor a and cosmic red - shift parameter $z=a_{0} / a-1$
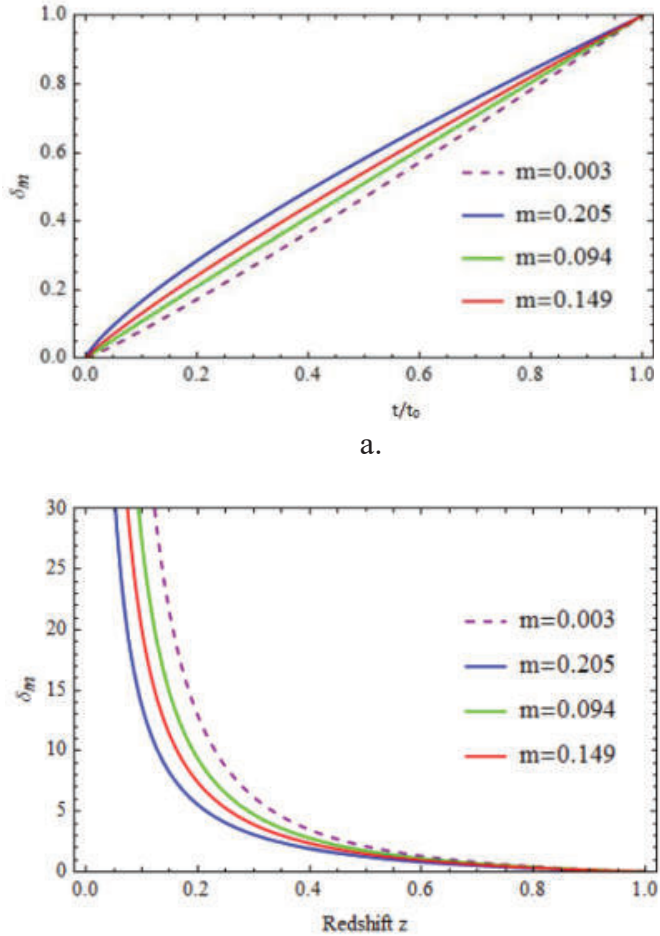

c.

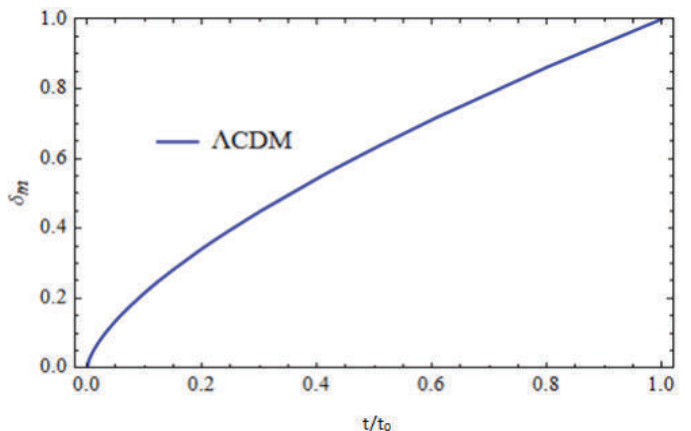

b.

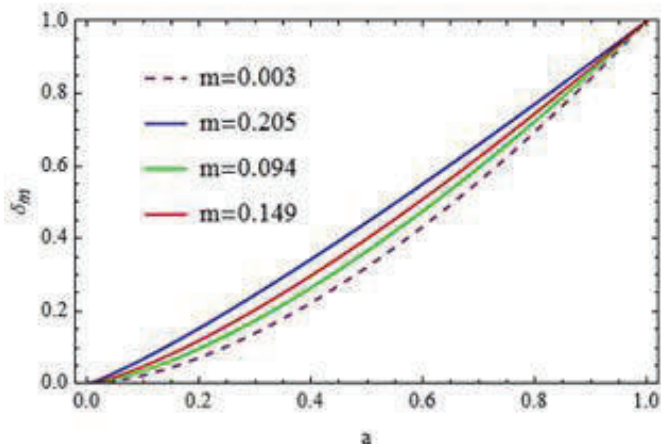

Figure1. Graphs with different values of $m$,

they are compared to that in the $\Lambda$ CDM standard cosmological model.

In figure.1a, as $\mathrm{m}$ approaches to zero, the graph is significantly different from that in the standard cosmological model; figure $1 \mathrm{~b}$ is the graph in the $\Lambda \mathrm{CDM}$ standard cosmological model; in figure $1 \mathrm{c}$, the perturbation $\delta$ as a function of redshift $\mathrm{z}$; in figure $1 \mathrm{~d}$, perturbation $\delta$ as a function of scale factor a(t).

4.2 The case $\frac{k^{2}}{a^{2} R} m<<1$ 
This condition is satisfied on distance scales that large structures of universe were made.

In this case, the equation (23) becomes

$$
\delta^{\prime \prime}+\left(\frac{1}{2}-\frac{3}{2} \omega_{e f f}\right) \delta^{\prime}-\frac{3}{2} \Omega_{m} G_{N} \delta=0
$$

Solving equation (33), we obtain

$$
\delta=c_{+} a^{\frac{1}{4}\left[-\sqrt{\frac{(4 m+1)^{2}-12 G_{N}\left(8 m^{2}+3 m-2\right)}{(m+1)^{2}}}-\frac{3 m}{m+1}-1\right]}+c_{-} a^{\frac{1}{4}\left[\sqrt{\frac{(4 m+1)^{2}-12 G_{N}\left(8 m^{2}+3 m-2\right)}{(m+1)^{2}}}-\frac{3 m}{m+1}-1\right]}
$$

The solution of the growth mode is

$$
\delta=a^{\frac{1}{4}\left[\sqrt{\frac{(4 m+1)^{2}-12 G_{N}\left(8 m^{2}+3 m-2\right)}{(m+1)^{2}}}-\frac{3 m}{m+1}-1\right]},
$$

or in the other form, it depends on cosmic time as follows

$$
\delta=t^{\frac{1}{6}\left[\sqrt{\frac{(4 m+1)^{2}-12 G_{N}\left(8 m^{2}+3 m-2\right)}{(m+1)^{2}}}-\frac{3 m}{m+1}-1\right]}
$$

When $m=0 \rightarrow \delta \propto a \propto t^{2 / 3}$.

Figure 2 shows cosmological perturbation in the growth mode on the scale factor a and cosmic redshift paramete
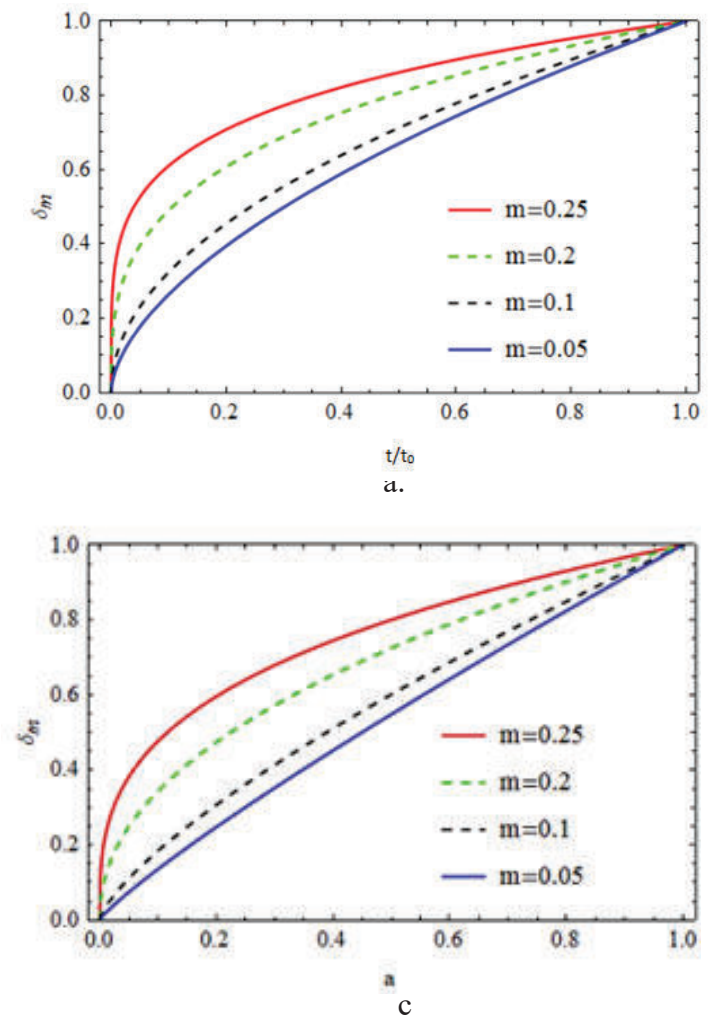

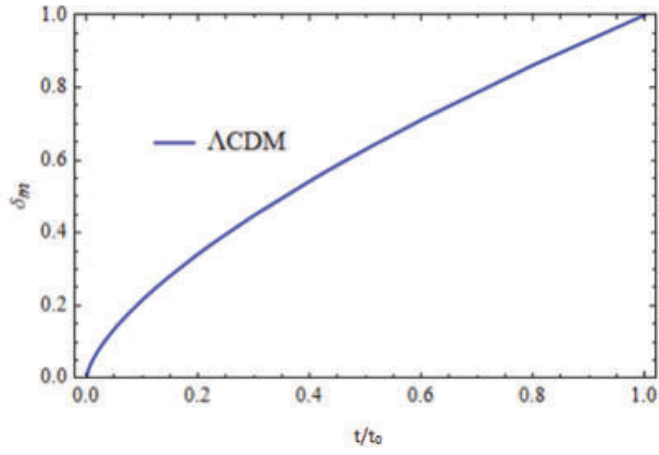

b.

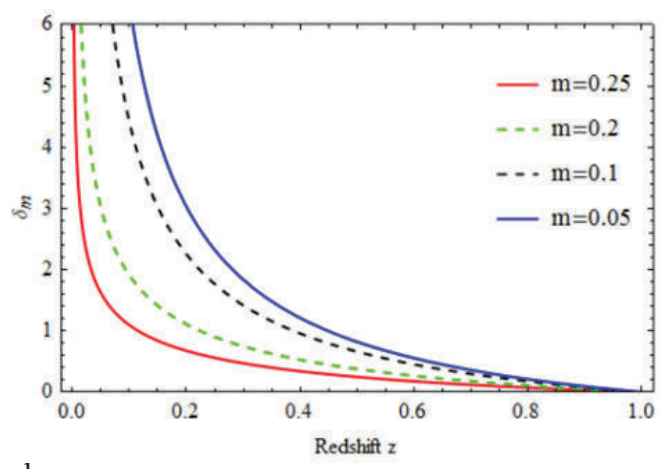

d.

Figure2. Graphs with different values of $\mathrm{m}$, they are compared to that in the $\Lambda \mathrm{CDM}$ standard cosmological model.

In the figure.2a, as $\mathrm{m}$ approaches to zero, the graph is the same with that in the standard cosmological model, when large $\mathrm{m}$, graph is significantly different from that in it; the figure $2 \mathrm{~b}$ is the graph in the $\Lambda \mathrm{CDM}$ standard cosmological model; in the figure $2 \mathrm{c}$, the perturbation $\delta$ as a function of redshift $\mathrm{z}$; in the figure.2d, the perturbation $\delta$ as a function of scale factor a. 


\section{The evaluation of the ratio $\Psi / \Phi$}

In this section we will re-evaluate the ratio of the scalar functions $\Psi / \Phi$ in this model and compare it to the experimental constraints. We have [24],

$$
\frac{\Psi}{\Phi}=\frac{1+4 \frac{k^{2}}{a^{2}} \frac{F_{R R}}{1+F_{R}}}{1+2 \frac{k^{2}}{a^{2}} \frac{F_{R R}}{1+F_{R}}},
$$

here $\mathrm{k}=0.01 \mathrm{hMpc}^{-1} \sim 10^{-25} \mathrm{~m}^{-1} ; \mathrm{h}=0.72 \pm 0.08, \mathrm{R} \sim 10^{-30} \mathrm{~m}^{-2}, \alpha=9 \times 10^{-75}$ [21].

In the present time, the scale factor $\mathrm{a}=1$, so we have in $\mathrm{f}(\mathrm{R})$ modified gravity of polynomial exponential form

$$
F_{R} \propto-\frac{\alpha}{R^{2}} ; \mathrm{F}_{R R} \propto \frac{2 \alpha}{R^{3}} ; \frac{F_{R R}}{1+F_{R}} \propto 2 \alpha R^{-3},
$$

therefore $\Psi / \Phi=1$.

In the early stages of the universe, the cosmic curvature is very large, the scale factor a is very small, if $\frac{k^{2}}{a^{2} R} m>>1$ with $m=\frac{R F_{R R}}{1+F_{R}}$, we have

$$
\frac{\Psi}{\Phi} \approx 2
$$

The experimental data when $\mathrm{k}=0.01 \mathrm{hMpc}^{-1}$ and $\mathrm{a}=1[24]$,

$$
1<\frac{\Psi}{\Phi} \leq 1.996
$$

The ratio $\Psi / \Phi$ in this model is within the allowable limits of the experiment.

\section{The evaluation of the ratio $G_{\text {eff }}$ / GN}

In this section, we calculate the ratio of the effective gravitational and the Newtonian constant and compare it with the constraints from experiments.

We have from formula (15),

$$
G_{e f f}=\frac{1}{1+F_{R}} \frac{1+4 \frac{k^{2}}{a^{2}} \frac{F_{R R}}{1+F_{R}}}{1+3 \frac{k^{2}}{a^{2}} \frac{F_{R R}}{1+F_{R}}} G_{N}
$$

In the present time [21,24], $R: 10^{-30} \mathrm{~m}^{-2}$, the scale factor $a=1$, we have

$$
\begin{aligned}
& F_{R}=\alpha\left[-3 \beta R^{2}-\frac{1}{R^{2}}-2(\beta-1) R+1\right] \cong-\frac{\alpha}{R^{2}} ; \\
& F_{R R}=2 \alpha\left[-\beta+\frac{1}{R^{3}}-3 \beta R+1\right] \cong \frac{2 \alpha}{R^{3}} ;
\end{aligned}
$$

with $\mathrm{k}=0.01 \mathrm{hMpc}^{-1} ; \mathrm{h}=0.72 \pm 0.08$.

with $\mathrm{k}=0.01 \mathrm{hMpc}^{-1} ; \mathrm{h}=0.72 \pm 0.08$.

Here $\alpha, \beta$ have the values [21],

we have

$$
\begin{gathered}
\alpha=9 \times 10^{-75} ; \beta=7.67 \times 10^{-15} ; \\
\frac{G_{e f f}}{G_{N}} \cong 1 .
\end{gathered}
$$


In the early stages of the universe, the cosmic curvature is very large, the scale factor a is very small.

When $\beta R=1 \rightarrow 1<R=10^{14} \mathrm{~m}^{-2}$, we have,

$$
\begin{aligned}
& F_{R}=\alpha\left[-3 \beta R^{2}-\frac{1}{R^{2}}-2(\beta-1) R+1\right] \cong-2 \alpha R \\
& F_{R R}=2 \alpha\left[-\beta+\frac{1}{R^{3}}-3 \beta R+1\right] \cong-6 \alpha \beta R
\end{aligned}
$$

If $\frac{k^{2}}{a^{2} R} m$ ? 1 with $m=\frac{R F_{R R}}{1+F_{R}}$, we have

$$
\frac{G_{e f f}}{G_{N}} \cong \frac{4}{3} \cong 1.33 \text {. }
$$

The constraint on the present time is [24],

$$
1 \leq \frac{G_{e f f}}{G_{N}} \leq 1.403 \text {. }
$$

The constraint in the recombination epochs is [25],

$$
\begin{aligned}
& \frac{G_{r e c}}{G_{o}}-1<1.9 \times 10^{-3}(95.45 \% \text { C.L. }) \\
& \frac{G_{r e c}}{G_{o}}-1<5.5 \times 10^{-3}(99.99 \% \text { C.L. })
\end{aligned}
$$

We see that the $\mathrm{G}_{\text {eff }} / \mathrm{G}_{\mathrm{N}}$ ratio changes according to the evolution of the universe, depending on the scale factor a, which has the largest deviation from unit at the early stage of the universe, but it almost coincides with unit in the current stage from this model.

\section{Conclusion}

In this article, we have used analytical method and numerical calculations to investigate the evolutionary aspects of perturbation of material density in $\mathrm{f}(\mathrm{R})$ modified gravity of exponential - polynomial form. The evolutionary aspects in this model are compared to that in the $\Lambda \mathrm{CDM}$ standard cosmological model. The results show a significant difference in the evolutionary aspect of perturbation in this model versus the standard model and can be used to distinguish it from the $\Lambda \mathrm{CDM}$ standard model and other models. The ratios $\Psi / \Phi$ and $\mathrm{G}_{\text {eff }} / \mathrm{G}_{\mathrm{N}}$ in this model are also within the allowable limits of experimental constraints.

\section{References}

[1] S. Perlmutter et al. (1999), "Measurements of $\Omega$ and $\Lambda$ from 42 High-Redshift Supernovae", Astrophys. J. 517, 565-586.

[2] P. Astier et al.(2006), "The Supernova Legacy Survey: measurement of $\Omega_{\mathrm{m}}, \Omega_{\Lambda}$ and w from the first year data set”, Astron. Astrophys. 447, 31-48.

[3] D. N. Spergel et al. [WMAP Collaboration].(2003), "First Year Wilkinson Microwave Anisotropy Probe (WMAP) Observations: Determination of Cosmological Parameters", Astrophys. J. Suppl. 148, 175-194.

[4] D. J. Eisenstein et al. [SDSS Collaboration](2005), "Detection of the Baryon Acoustic Peak in the Large-Scale Correlation Function of SDSS Luminous Red Galaxies”, Astrophys.J. 633, 560-574.

[5] V. Sahni and A. A. Starobinsky.(2000), "The Case for a Positive Cosmological Lambda-term", Int. J. Mod. Phys. D 9, 373-444.

[6] E. J. Copeland, M. Sami and S. Tsujikawa.(2006), "Dynamics of dark energy”, Int. J. Mod.Phys. D 15, 17531936.

[7] L. H. Ford.(1987), “Cosmological-constant damping by unstable scalar fields”, Phys. Rev. D 35, 2339-2344. 
[8] B. Ratra and P. J. E. Peebles.(1988), “Cosmological consequences of a rolling homogeneous scalar field”, Phys. Rev. D 37, 3406-3427.

[9] T. Chiba, N. Sugiyama and T. Nakamura.(1997), “Cosmology with X-matter”, Mon. Not. Roy. Astron. Soc. 289, L5-L9.

[10] P. G. Ferreira and M. Joyce.(1997), "Structure Formation with a Self-Tuning Scalar Field”, Phys. Rev. Lett. 79, 4740- 4743

[11] E. J. Copeland, A. R. Liddle and D. Wands.(1998), "Exponential potentials and cosmological scaling solutions", Phys. Rev. D 57, 4686- 4690.

[12] R. R. Caldwell, R. Dave and P. J. Steinhardt.(1998), “Cosmological Imprint of an Energy Component with General Equation of State", Phys. Rev. Lett. 80, 1582- 1585.

[13] I. Zlatev, L. M. Wang and P. J. Steinhardt.(1999), “Quintessence, Cosmic Coincidence, and the Cosmological Constant", Phys. Rev. Lett. 82, 896-899.

[14] T. Chiba, T. Okabe and M. Yamaguchi.(2000), "Kinetically driven quintessence", Phys. Rev. D 62, 0235111- 023511-8.

[15] C. Armendariz-Picon, V. F. Mukhanov and P. J. Steinhardt.(2000), "Dynamical Solution to the Problem of a Small Cosmological Constant and Late-Time Cosmic Acceleration", Phys. Rev. Lett. 85, 4438-4441.

[16] A. Y. Kamenshchik, U. Moschella and V. Pasquier.(2001), “An alternative to quintessence”, Phys. Lett. B 511, 265-268.

[17] T. P. Sotiriou and V. Faraoni.(2010), "f(R) theories of gravity”, Rev. Mod. Phys. 82, 451-497.

[18] Hayato Motohashi, Alexei A. Starobinsky and Jun'ichi Yokoyama.(2009), “Analytic solution for matter density perturbations in a class of viable cosmological $\mathrm{f}(\mathrm{R})$ models", arXiv: 0905.0730v3.

[19] Boisseau, B.; Esposito-Farese, G.; Polarski, D.; Starobinsky, A.A.(2000), "Reconstruction of a Scalar-Tensor Theory of Gravity in an Accelerating Universe", Phys. Rev. Lett. 85, 2236-2239.

[20] Vo Van On, and Tran Trong Nguyen.(2015), "Some Scenarios of Cosmological Revolution in Polynomial Exponential f(R) Modified Theory of Gravity", Chinese Journal of Physic 53, 3, 060101-1-060101-10.

[21] Vo Van On, Nguyen Ngoc.(2018), "Chameleon mechanism in $\mathrm{f}(\mathrm{R})$ modified gravitation model of polynomialexponential form”, Journal of Physics: Conf. Series 1034, 012002-1- 012002-9.

[22] Vo Van On, Truong Huu Nghi.(2018), Report in National Conference on Theoretical Physics43, ICISE Quynhon.

[23] T. Damour and K. Nordtvedt.(1993), "Tensor-scalar cosmological models and their relaxation toward general relativity”, Phys. Rev. D 48, 3436-3450.

[24] Wei-Ting Lin; Je-An Gu and Pisin Chen.(2010), “Cosmological and Solar-System Tests of f (R) Modified Gravity", arXiv: 1009. 3488.

[25] Ooba, J.; Ichiki, K.; Chiba, T.; Sugiyama, N.(2017), “Cosmological constraints on scalar-tensor gravity and the variation of the gravitational constant”, arXiv: 1702.00742. 